\title{
Detection and Isolation Method for Operator Failure by Unknown Input Observer
}

\author{
+ Hwan-Seong Kim · Seung-Min Kim* \\ + Dept. of Logistics, Korea Maritime University, Busan 606-791 Korea \\ * Dept. of Mechatronics Examination, The Korean Intellectual Property Office, Daejeon, Korea
}

\begin{abstract}
In this paper, a fault detection method for operator failures using the observation technique is proposed. The suggested algorithm is extended using the conventional sensor/actuator fault detection method. First, it is assumed that operator failure affects human work operations, as it is an external input signal. With this assumption, a human work model with operator failure is suggested. Second, an unknown input observer with proportional and integral gains is introduced. The characteristic of this observer of estimating an external signal without an exact input is shown, and the conditions for the detection of an operator failure are proposed. Finally, by simulating the container crane operations, it is verified that the observer can accurately detect an operator failure and estimate its magnitude from the given internal signal.
\end{abstract}

Key words : Operator failure, Observer, Model, Fault detection, Isolation, Container crane

\section{Introduction}

Nowadays, an increasing number of industrial systems are equipped with high-end functions and automation, especially modern port systems and container terminal systems. In these systems, an operator generally runs most tasks. During the work process, however, the operator might commit some mistakes; and if s/he works for an extended period, s/he may cause an accident. It is thus necessary to develop monitoring and diagnostic systems for human operation during the work process.

The generic approach to the assessment of human error has three steps. The first step is human error identification, to reduce human error in systems. The second step quantifies the errors that may be needed to construct a safety case. The final step develops error databases to reduce human error.

These steps are related to the identification of a model of human behavior that will enable the design of satisfactory manually controlled systems. Such model of human behavior is identified by analyzing the neuro-muscular characteristics of the human operator. Shinners(1947), Malek et al.(1988), Yehia(1995), and Martens(1999) have mentioned some of the studies that employed these steps. Their approach considered the human operator an inanimate servomechanism with well-defined inputs and outputs.

Over the years, the evolution of the control-theory paradigm for the human controller or operator has paralleled the development of new synthesis techniques in feedback control. Thus, optimal control models (OCMs) of the human operator have appeared as linear quadratic Gaussian (LQG) control system design techniques. Fuzzy controller models and $\mathrm{H}$ infinity models of the human operator closely followed the appearance of these design techniques (Bryson and Ho, 1975; Franklin et. al., 1998; Levine, 1996; Skogestad and Postlethwaite, 1996; Antsaklis and Anthony, 1997; Zhou and Doyle, 1998).

These models seek to account for many subsystem characteristics of the human operator by assigning transfer functions to the different subsystems involved. These subsystems and their interconnections are postulated on the basis of physiologically isomorphic considerations.

Shinners(1947) first introduced the application of time-series analysis to this problem. He developed autoregressive moving-average (ARMA) models of data collected from human operators who were involved in compensatory tracking experiments using band-limited white noise inputs (bandwidth $=1.5[\mathrm{~Hz}]$ ). His results showed that all the operators exhibited a time delay of $0.2[\mathrm{~s}]$ and that the discrete transfer functions that represented their dynamics had one zero and two poles. Based on the analysis of model residuals, he concluded that the human operator is a generator of seasonal (rhythmic) characteristics during tracking of random inputs.

Generally, an important step in the modeling of human operator (HO) dynamics using the time--series approach is

\footnotetext{
+ Corresponding Author : kimhs@hhu.ac.kr, +82-51-410-4334

* kipo0070@kipo.go.kr
} 
the identification of the model order (i.e., the number of poles and zeros in the model). Several model order determination criteria that are commonly used in systems identification have been critically evaluated in terms of their ability to estimate the order of a simulated autoregressive model (AR) with parameters obtained from $\mathrm{HO}$ input-output data during a compensatory tracking experiment (Sinha and Kuszta, 1983; Glass and Wong, 1988; Efe and Kaynak, 1999; Ljung, 1999).

On the other hand, the detection and isolation of actuator and/or sensor failures have received considerable attention during the last two decades in both theoretical researches and practical applications (Clark, 1978; Frank and Keller, 1980; Park and Lee, 1999). Although there have been several studies on fault detection and isolation (FDI) observers (Viswanadham and Srichander, 1987; Hou and Muller, 1994; Wang and Daley, 1996) and residual generators (Garcia and Frank, 1999), such studies were concerned with only the partial detection and isolation of actuator failures or sensor failures. In the case of the FDI observer, multiple observers have been used to determine the localization of faulty elements in the process, where as many unknown-input observers were constructed as the number of inputs (Viswanadham and Srichander, 1987).

Recently, a fault diagnosis method of detecting and isolating actuator and sensor failures, including the magnitude of the fault, has been proposed using multiple proportional integral (PI) observers (Kim et. al., 1997). It is based on the intensive use of knowledge on the characteristics of PI observers, which estimate and cancel step actuator failures.

In this paper, a fault detection method for operator failures using the observation technique is proposed. The suggested algorithm is extended using the conventional sensor/actuator fault detection method. First, it is assumed that operator failure affects human work operations, as it is an external input signal. With this assumption, a human work model with operator failure is suggested.

Second, an unknown input observer with proportional and integral gains is introduced. The characteristic of this observer of estimating an external signal without an exact input is shown, and the conditions for the detection of an operator failure are proposed.

Finally, by simulating the container crane operations, it is verified that the observer can accurately detect an operator failure and estimate its magnitude from the given internal signal when the human model from previous researches (Kim et. al., 2005) is used.

\section{Design of observer}

\subsection{Problem statements}

We assume that an operator work model is described by linear time-invariant model as:

$$
\begin{aligned}
& \dot{x}(t)=A x(t)+B u(t)+D d(t) \\
& y(t)=C x(t)
\end{aligned}
$$

where $x(t) \in \mathrm{R}^{n}$ denotes state vector, $u(t) \in \mathrm{R}^{m}$ input vector, $y(t) \in \mathrm{R}^{\mathrm{p}}$ output vector, and $d(t) \in \mathrm{R}^{q}$ disturbance vector, respectively. Matrices $A, B, C$ and $D$ are appropriate dimensions and fair $(C, A)$ is observable.

In Eq. (1), the input $u(t)$ includes all the control information that was recognized during the operations. The output $y(t)$ denotes the operation outputs, such as the joystick and/or button signals, under the operator's control resulting from learning, experiments, knowledge, know-how, etc. The disturbance $d(t)$ includes uncertainties from the changes in the work environment, the changes in the operator's work conditions, and the changes in the weather. In this paper, it is assumed that an operator has committed a mistake from wrong information, and that this has resulted in operator failure. Due to such mistake, the system would not work normally. In the operator work model, the wrong information will be added to the input terms. To do this, normal condition input $u(t)$ and abnormal condition input $\bar{u}(t)$ are defined. The abnormal condition input $\bar{u}(t)$ includes the wrong information. From this definition, the input term in Eq. (1a) will be modified as:

$$
\bar{u}(t)=u(t)+a(t)
$$

where $a(t) \in \mathrm{R}^{\mathrm{m}}$ denotes the wrong information which make the mistake by operator and it will be added to input term in (1a).

By substituting Eq. (2) into Eq. (1), we obtain the following model.

$$
\begin{aligned}
& \dot{x}(t)=A x(t)+B u(t)+B a(t)+D d(t) \\
& y(t)=C x(t)
\end{aligned}
$$

The aim of this paper is to detect and isolate the operator failures $a(t)$ that is included to the input term by using observer techniques perfectly. 


\subsection{Structure of Observer}

In this paper, we deal with an observer include proportional and integral gains. The structure of observer is given as follows:

$$
\begin{aligned}
& \dot{z}(t)=\hat{A} z(t)+\hat{B} y(t)+\hat{J} u(t)+\hat{H} \omega(t) \\
& \dot{x}(t)=\hat{C} z(t)+\hat{D} y(t) \\
& \dot{\omega}(t)=y(t)-\hat{C} \hat{x}(t)
\end{aligned}
$$

where $z(t) \in \mathrm{R}^{n}$ denotes the observer state vector, $\hat{x}(t) \in \mathrm{R}^{n}$ the estimated state vector, $\omega(t) \in R^{p}$ output error, and matrices $\hat{A}, \hat{B}, \hat{C}, \hat{D}, \hat{H}$ and $\hat{J}$ are appropriate dimension for observer.

Definition 1: For operator work system in Eq. (1), the system Eq. (4) is said to be an observer if and only if

$$
\begin{aligned}
& \lim _{t \rightarrow \infty} e(t)=0, \forall z\left(0_{-}\right), x\left(0_{-}\right), u(\cdot) \\
& \lim _{t \rightarrow \infty} \omega(t)=0, \forall \omega\left(0_{-}\right)
\end{aligned}
$$

where $e(t)=x(t)-\hat{x}(t)$ denotes the estimated state error.

Under the Definition 1, we have the following theorem which describes the relation between the system and the observer.

Theorem 1: The system Eq. (4) is an observer for the system Eq. (3) with an unknown external input if

$$
\operatorname{Re} \lambda_{i}\left[\begin{array}{cc}
\hat{A} & \hat{H} \\
-\hat{C} \hat{C} & 0
\end{array}\right]<0, i=1, \cdots, n+p
$$

and there exist a matrix $U \in R^{n \times n}$ such that

$$
\begin{aligned}
& \hat{A} U+\hat{B} C=U A \\
& \hat{J}=U B \\
& \hat{C} U+\hat{D} C=I_{n} \\
& U D=0
\end{aligned}
$$

where $\operatorname{Re} \lambda_{i}[\cdot]$ denotes the eigenvalues of matrix.

Proof : let us define an estimation error $\zeta(t)$ as

$$
\zeta(t)=z(t)-U x(t)
$$

Then, the dynamics of this error Eq. (10) obeys

$$
\begin{aligned}
\zeta(t)= & \hat{A} \zeta(t)+(\hat{A} U+\hat{B} C-U A) x(t) \\
& -U D d(t)+(\hat{J}-U B) u(t)+\hat{H} \omega(t)
\end{aligned}
$$

Also, Eq. (4b) leads to

$$
\begin{aligned}
& \hat{x}(t)=\hat{C} \zeta(t)+(\hat{C} U+\hat{D} C)_{x}(t) \\
& \dot{\omega}(t)=C(x(t)-\hat{x}(t))
\end{aligned}
$$

By substituting Eqns. (6) - (9) into Eqns. (11) - (12), we have

$$
\begin{aligned}
& \dot{\zeta}(t)=\hat{A} \zeta(t)+\hat{H} \omega(t) \\
& \hat{x}(t)=\hat{C} \zeta(t)+x(t) \\
& \dot{\omega}(t)=-\hat{C} \hat{C}(t)
\end{aligned}
$$

or

$$
\begin{aligned}
& {\left[\begin{array}{c}
\dot{\zeta}(t) \\
\dot{\omega}(t)
\end{array}\right]=\left[\begin{array}{cc}
\hat{A} & \hat{H} \\
-\hat{C} \hat{C} & 0
\end{array}\right]\left[\begin{array}{c}
\zeta(t) \\
\omega(t)
\end{array}\right]} \\
& \hat{x}(t)=\hat{C} \zeta(t)+x(t)
\end{aligned}
$$

Thus, under the condition Eq. (5), $\omega(t) \rightarrow 0$ and $e(t) \rightarrow 0$ $(t \rightarrow \infty)$. So, this proof is completed.

The problem of designing the observer for the operator work model with unknown disturbance input is reduced to find the matrices $\hat{A}, \hat{B}, \hat{C}, \hat{D}, \hat{H}, \hat{J}$ and $U$ such that the conditions of Theorem 1 are satisfied.

In the next section, we will show the fault detection characteristic and the realization condition for the observer.

\section{Detection of operator failure}

\subsection{Characteristic of operator failure detection and isolation}

In this section, we describe the characteristics of fault detection for operator mistake. For this, consider the dynamics of system with the operator failure and interconnected unknown disturbance Eq. (3).

By using the estimation error Eq. (10), we have the following error dynamics from Eq. (3) and Eq. (4).

$$
\begin{aligned}
\dot{\zeta}(t) & =\hat{A} \zeta(t)+(\hat{A} U+\hat{B} C-U A) x(t) \\
& -U D d(t)+(\hat{J}-U B) u(t)+\hat{H} \omega(t)-U B a(t) \\
\hat{x}(t) & =\hat{C} \zeta(t)+(\hat{C} U+\hat{D} C) x(t)
\end{aligned}
$$




$$
\dot{\omega}(t)=C(x(t)-\hat{x}(t))
$$

By applying Theorem 1, we have

$$
\begin{aligned}
& \dot{\zeta}(t)=\hat{A} \zeta(t)+\hat{H} \omega(t)-U B a(t) \\
& \hat{x}(t)=\hat{C} \zeta(t)+x(t) \\
& \dot{\omega}(t)=-\hat{C} \hat{C} \zeta(t)
\end{aligned}
$$

Let $\xi(t)$ be defined as

$$
\xi(t)=\omega(t)-a(t)
$$

Under the assumption of step operator failures (because the operator failure does not change frequently) $\dot{a}(t)=0$, we obtain

$$
\dot{\xi}(t)=\dot{\omega}(t)=-\hat{C} \hat{C}(t)
$$

If we design the matrix $\hat{H}$ as

$$
\hat{H}=U B
$$

Then, we get

$$
\begin{aligned}
& \dot{\zeta}(t)=\hat{A} \zeta(t)+\hat{H} \xi(t) \\
& \dot{\xi}(t)=-\hat{C} \hat{C} \zeta(t)
\end{aligned}
$$

or

$$
\left[\begin{array}{l}
\dot{\zeta}(t) \\
\dot{\xi}(t)
\end{array}\right]=\left[\begin{array}{cc}
\hat{A} & \hat{H} \\
-\hat{C} \vec{C} & 0
\end{array}\right]\left[\begin{array}{l}
\zeta(t) \\
\xi(t)
\end{array}\right]
$$

Under the condition of Theorem $1, \quad \zeta(t), \xi(t) \rightarrow 0$ $(t \rightarrow \infty)$. Therefore, the operator failure $a(t)$ can be estimated as

$$
\hat{a}(t)=\omega(t)
$$

From the above statements, the magnitude of operator failure can be estimated and the failure is effectively isolated in the system with unknown external disturbance. Based on the above statement, we have the following lemma.

Lemma 1: The operator failure is detected and isolated effectively by using observer Eq. (4) for operator work model with unknown external disturbance Eq. (3), if the condition of Theorem 1 is satisfied.

\subsection{Realization of observer}

To realize the observer, we let $\hat{C}=I_{n}$ for simplicity. Then, from Eq. (8) we obtain

$$
U=I_{n}-\hat{D} C
$$

By substitution of Eq. (23) into Eq. (6), we have

$$
\begin{aligned}
& \hat{A}=U A-K C \\
& \hat{B}=\hat{A} \hat{D}+K
\end{aligned}
$$

where, matrix $K$ can be designed arbitrarily.

Substituting Eq. (27) into Eq. (9), we have

$$
\hat{D} C D=D
$$

In order to guarantee the matrix $\hat{D}$ satisfying Eq. (30), the following condition should be hold.

$$
\operatorname{rank} C D=\operatorname{rank} D=q
$$

The condition Eq. (30) requires that $p \geq q$ i.e., the number of measured output must be greater than or equal to that of the external disturbance input.

The general solution Eq. (30) can be obtained as

$$
\hat{D}=D(C D)^{+}+G\left(I_{p}-C D(C D)^{+}\right)
$$

where the superscript + indicates the generalized inversion and $G$ is an arbitrary matrix.

By substituting Eq. (32) into Eq. (27), we can get

$$
U=\left(I_{n}-G C\right) I_{n}-D(C D)^{+} C
$$

From the above equation, there exists a matrix $G$, which make $\left(I_{n}-G C\right)$ non-singular, and then the $\operatorname{rank} U=n-q$.

Since rank $D=q$, there exists the left inverse of matrix $D$, i.e., $D^{+} D=I_{q}$.

Under the condition of rank $U=n-q$, we have $\operatorname{Ker} U \cup \operatorname{Ker} D^{+}=0$, i.e.,

$$
\operatorname{rank}\left[\begin{array}{c}
U \\
D^{+}
\end{array}\right]=n
$$

Then, we have the following relation

$$
\operatorname{rank}\left[\begin{array}{cc}
s I_{n}-A & D \\
C & 0
\end{array}\right]=q+\operatorname{rank}\left[\begin{array}{c}
s I_{n}-U A \\
C
\end{array}\right]
$$

Consequently, for $\forall s \in \mathrm{C}$, where $\mathrm{C}$ denotes the complex space,

$$
\operatorname{rank}\left[\begin{array}{cc}
s I_{n}-A & D \\
C & 0
\end{array}\right]=n+q, \quad \forall s \in \mathrm{C}
$$


which means that the invariant zeros of the system ( $A, D, C, 0)$ must be stable. From the above statements, we summarize the following theorem.

Theorem 2: The observer Eq. (4) for the operator work model Eq. (3) can be realized if

(i) $\operatorname{rank} C D=\operatorname{rank} D=q$

(ii) $\operatorname{rank}\left[\begin{array}{cc}s I_{n}-A & D \\ C & 0\end{array}\right]=n+q, \quad \forall s \in \mathrm{C}$

Remark 1: The eigenvalues of the observer depend upon the arbitrary matrix $G$ and the matrix $K$ significantly.

\section{Simulation and results}

\section{1 Operator work model}

In the simulation, previous researches (Kim et. al., 2005) were referred to determine the operator work model where the container crane simulator was used. To obtain the operator work model, the human operation model for the container crane system had to be constructed. Fig. 1 shows the basic human work model that represents the container transportation from its initial position to its reference position, where the container should avoid the given obstacles.

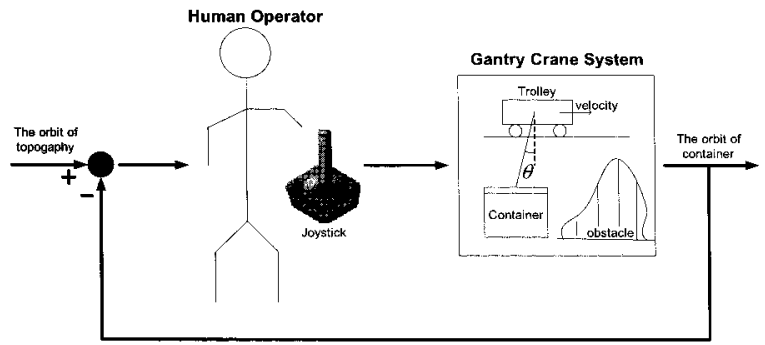

Fig. 1 Representation of human operation for container crane system

In Fig. 1, the operator operates the joystick to control the trolley position and the hoist cable length by motors with the motor inverters, respectively. In this case, the operator can watch the container movement and its sway angle. If s/he recognizes an error between the desired and actual positions of the container, s/he can decide to control such error by using a joystick to compensate for the error. The signals from the joystick from the horizontal and vertical directions are proportional to the rate of change of the forces on the trolley and the cable, respectively.
Using the container crane operation, the human operations data such as the joystick angle; the container crane operations such as the trolley position, the container position, the sway angle, the cable length, etc.; and the desired container transportation trajectory must be determined.

To identify the human work model for the container crane system, the input and output factors were distinguished from the above mentioned data. Here, it was assumed that the desired container trajectory would be given using the optimal control method or other procedures.

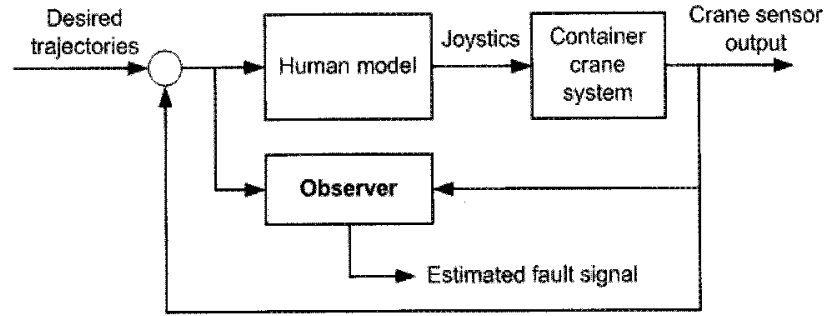

Fig. 2 A block diagram for estimating human fault

However for estimating the human failure, in this paper, the input term is given as the real trolley position and the sway angle. The joystick angles for the trolley and hoist control are given as output terms. To do this, a block diagram for the human failure detect model was made and is shown in Fig. 2.

In the simulation, three work trajectory paths for one person were made and human operations data were collected with an $80[\mathrm{~ms}]$ sampling time.

\subsection{Design of observer}

We consider the human model Eq. (3) with matrices as

$$
\begin{aligned}
& A=\left[\begin{array}{rrr}
-11.937 & -1.3040 & -18.713 \\
0.4879 & -0.1736 & 0.0626 \\
3.4741 & -0.3493 & -8.8390
\end{array}\right], B=\left[\begin{array}{rr}
-3.4475 & 8.7215 \\
-0.7417 & 1.4307 \\
-16.3598 & 27.2699
\end{array}\right] \\
& D=\left[\begin{array}{l}
1 \\
0 \\
0
\end{array}\right], \quad C=10^{3} \times\left[\begin{array}{rrr}
-0.7176 & -0.0194 & -0.4918 \\
0.0940 & 1.9938 & -0.1791
\end{array}\right]
\end{aligned}
$$

To design the observer, the matrix $\hat{D}$ is calculated from Eq. (32) with $\hat{C}=I_{3}$ and $G$ as follows

$\hat{D}=\left[\begin{array}{rr}-0.0428 & -0.3159 \\ \mathbf{0 . 0 1 5 5} & 0.1187 \\ 0.0293 & 0.2237\end{array}\right]$ and $G=\left[\begin{array}{rr}0.1290 & -0.3384 \\ \mathbf{0 . 2 9 8 5} & 0.0816 \\ 0.1510 & 0.2078\end{array}\right]$

Then, the matrix $U$ is obtained from Eq. (33) as 


$$
U=\left[\begin{array}{rrr}
0 & 629.0040 & -77.6232 \\
0 & -235.2972 & 28.9019 \\
0 & -445.5262 & 55.4931
\end{array}\right]
$$

And we can easily see $U D=0$. The other observer matrices $\hat{A}, \hat{B}, \hat{H}$ and $\hat{J}$ are calculated from Eq. (22), Eq. (28) and Eq. (29) with arbitrary given matrix $K$ as

$$
\begin{aligned}
& \hat{A}=\left[\begin{array}{rrr}
29.9261 & -56.2127 & 717.2587 \\
9.0532 & 82.7675 & -260.3952 \\
-47.6147 & 51.5765 & -533.4423
\end{array}\right] \\
& \hat{B}=\left[\begin{array}{rr}
18.8573 & 144.3433 \\
-6.7036 & -51.3251 \\
-12.8286 & -98.1885
\end{array}\right] \\
& \hat{J}=\hat{H}=U B=10^{3} \times\left[\begin{array}{rr}
0.8034 & -1.2169 \\
-0.2983 & 0.4515 \\
-0.5774 & 0.8759
\end{array}\right]
\end{aligned}
$$

with

$$
K=\left[\begin{array}{rr}
-0.0119 & -0.0131 \\
0.0293 & -0.0258 \\
-0.0317 & 0.0029
\end{array}\right]
$$

\subsection{Results and considerations}

The response results for the container crane system in the simulation are shown in Fig. 3 for two cycles of work operations.
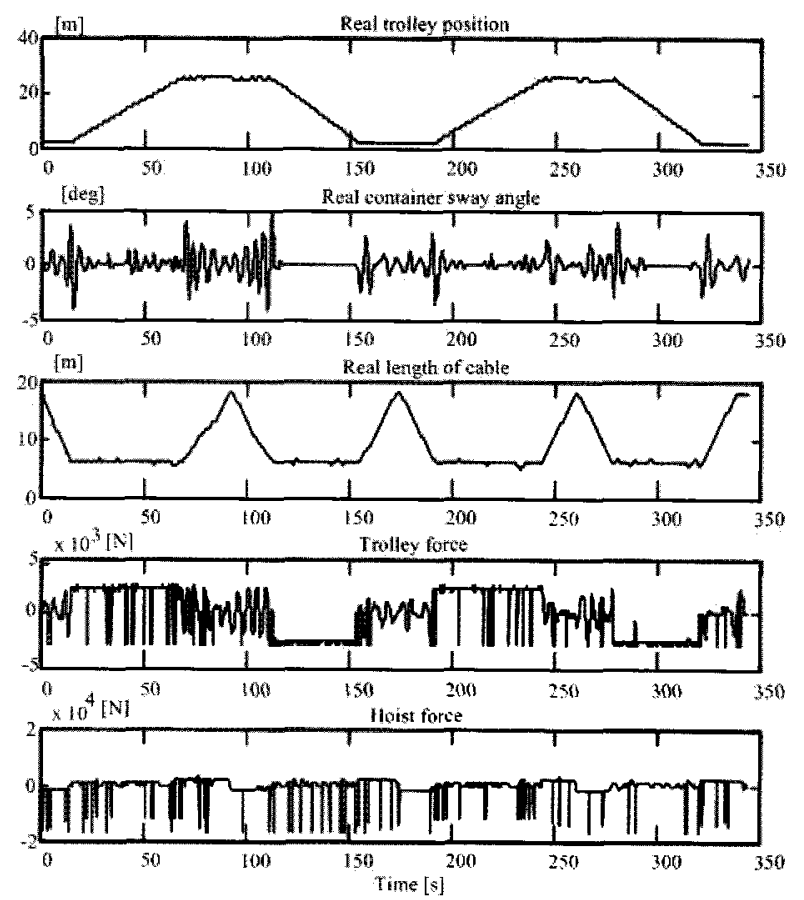

Fig. 3 Reponses of container crane in normal case
To verify the suggested observer so as to estimate the operator failure for its detection and isolation, it was assumed that the operator made a mistake in handling the trolley joystick for 5 seconds at $50[\mathrm{~s}]$.
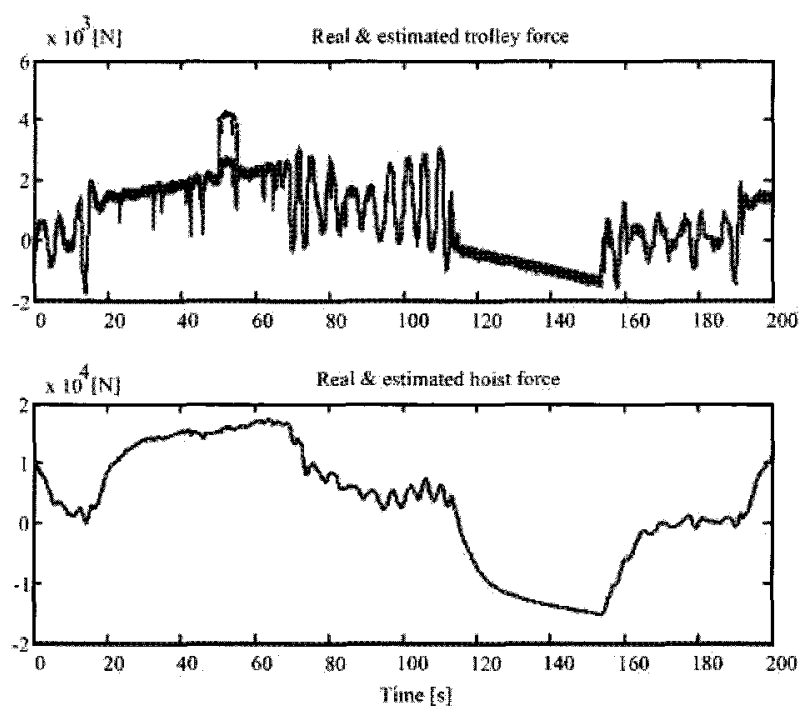

Fig. 4 Reponses of container crane with human fault

The simulation results for this case are shown in Fig. 4, where the upper and lower figures show the simulation results for the trolley and hoist operations, respectively.

As shown in Fig. 4, the hoist values are almost the same, but the trolley values do not match, especially at 50[s]. This shows that the operator failure appeared at $50[\mathrm{~s}]$.

To detect the exact operator failure, the observer signal $\omega(t)$ in Eq. (26) was taken. It is shown in Fig. 5. The estimated operator failures with the trolley and the hoist are shown in Fig. 5. The operator failure was detected for the trolley part at $50[\mathrm{~s}]$.
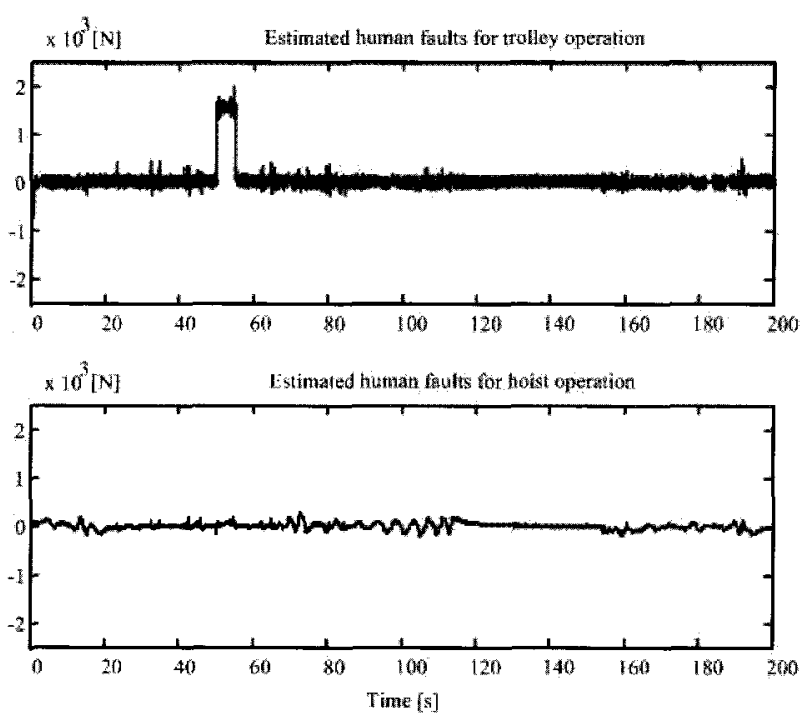

Fig. 5 Estimated human faults for trolley and hoist 
From the results, it was verified that the suggested observer had an effect on the detection and isolation of operator failure.

\section{Conclusions}

In this paper, a fault detection and isolation method for operator failures using the observation technique is suggested. The suggested algorithm was derived from the results of previous researches on the sensor/actuator fault detection method.

First, it was assumed that operator failure affects human work operations as an external input signal. With this assumption, a human work model with operator failure was suggested.

Second, an unknown input observer with proportional and integral gains was introduced. The characteristic of this observer of estimating an external signal without an exact input was shown, and the conditions for the detection of the operator failure were proposed. Finally, by simulating the container crane operations, it was verified that the observer could accurately detect operator failure and estimate its magnitude from the given internal signal.

Future researches should study detailed operator failures such as due to emotions and stress, including stress from environmental changes.

\section{Acknowledgements}

The first author would like to acknowledge the financial assistance provided by Korea Research Foundation for the 21st century.

\section{References}

[1] Antsaklis, P. J. and Anthony, N. M. (1997), "Linear Systems", McGraw-Hill.

[2] Bryson, A. E. and Ho, J. Y. C. (1975), "Applied Optimal Control: Optimisation, Estimation, and Control", John Wiley\&Sons, New York.

[3] Clark, R. N. (1978), "Instrument fault detection", IEEE Trans. Aero. and Electr. Syst., AES-14, pp. 456-465.

[4] Efe, M. O. and Kaynak, O. (1999), "Neural-fuzzy approaches for identification and control of non-linear Systems", Proceedings of the IEEE International Symposium on Industrial Electronics, pp. TU2-TU11.

[5] Frank, P. M. and Keller, L. (1980), "Sensitivity discriminating observer design for instrument failure detection", IEEE Trans. Aero. and Elect. Syst., AES-16, pp. $456-465$.

[6] Franklin, G. F., Powell, J. D., and Workman, M. (1998), "Digital Control of Dynamic Systems", Addison Wesley.

[7] Garcia, E. A. and Frank, P. M. (1999), "A novel design of structured observer-based residuals for FDI", Proceedings of the American Control Conference, pp. 1341-1345.

[8] Glass, B. J. and Wong, C. M. (1988), "A knowledgebased approach to identification and adaptation in dynamical systems control", Proceedings of the 27th IEEE Conference on Decision and Control, pp. 881-886.

[9] Hou, M. and Muller, P. C. (1994), "Fault detection and isolation observers", Int. J. Control, 60(5), pp. 827-846.

[10] Kim, H. S., Kim, S. B., and Kawaji, S. (1997),"Fault detection and isolation of system using multiple PI observers", Journal of the Korean Society of Precision Engineering, 14(2), pp. 41-47.

[11] Kim, H. S., Tran, N. H. S., and Kim, S. H. (2005), "Design of human works model for gantry crane system", International Journal of Navigation and Port Research, 29(2), pp. 135-140.

[12] Levine, W.S. (1996), "The Control Handbook", IEEE Press, Vol. II.

[13] Ljung, L. (1999), "System Identification: Theory for User", Prentice Hall PTR.

[14] Malek, A. A. and Marmarelis, V. Z. (1988), "Modelling of task-dependent characteristics of human operator dynamics pursuit manual tracking", IEEE Trans. Syst. Man, and Cybernetics, 18(1), pp. 163-172.

[15] Martens, D. (1999), "Neural networks as a tool for the assessment of human pilot behaviour in wind shear", Aerospace Science and Technology, 3(1), pp. 39-48.

[16] Park, J. P. and Lee, C. W. (1999), "Diagnosis of faults in rolling element bearings by using directional spectra of vibration signals", KSME Int. Journal, 13(1), pp. 63-73.

[17] Shinners, S. M. (1947), "Modelling of human operator performance utilizing time series analysis", IEEE Trans. Syst. Man Sybern., SMC-4(5), pp. 446-458.

[18] Sinha, N. K. and Kuszta, B. (1983), "Modeling and Identification of Dynamic Systems", Van Nostr and Reinhold Company, New York.

[19] Skogestad, S. and Postlethwaite, I. (1996), "Multivariable Feedback Control, Analysis and Design", John Wiley \& Sons, New York.

[20] Viswanadham, N. and Srichander, R. (1987), "Fault 
detection using unknown-input observer", Control-Theory and Advanced Technology, 3(2), pp. 91-101.

[21] Wang, H. and Daley, S. (1996), "Actuator fault diagnosis: an adaptive observer based technique", IEEE Trans. on Automatic Control, 41(7), pp. 1073-1078.

[22] Yehia, M. E. (1995), "Human operator behaviour modelling using non-linear identification techniques", Proceeding of Instrumentation and Measurement Technology Conference, pp 211-216.
[23] Zhou, K, and Doyle, J. C. (1998), "Essentials of Robust Control", Prentice Hall.

Received 5 March 2008

Accepted 31 March 2008 